\title{
Phantom Harmonic Gradient Estimators for Nonpreemptive Priority Queueing Systems
}

\author{
Felisa J. Vázquez-Abad* \\ Department of Computer Science and Operations Research \\ University of Montreal, Montreal, Canada H3C 3J7 \\ Email: vazquez@iro.umontreal.ca \\ Sheldon H. Jacobson ${ }^{\dagger}$ \\ Department of Mechanical and Industrial Engineering \\ University of Illinois at Urbana-Champaign, Urbana, IL 61801 \\ Email: shj@uiuc.edu
}

Submitted to INFORMS Journal on Computing, February 1999.

Revised October 30, 2000

\begin{abstract}
This paper presents a new gradient estimator for the steady-state expected sojourn (system) time in a nonpreemptive priority queueing system. The estimator uses the concept of a phantom system, together with the basic ideas in harmonic gradient estimation, to develop a single simulation run estimator, termed the phantom harmonic gradient estimator. The estimator is shown to be strongly consistent and strongly consistent in the average sense as the sample size grows. An upper bound for the variance of the PHG estimator is presented. This bound is used to show that under mild conditions, the variance of the PHG estimator tends to zero as both the number of phantom systems and the sample size approach infinity. A variance reduction technique that simultaneously uses both common and antithetic random numbers is presented. Computational results on several non-preemptive queueing systems illustrate the effectiveness of the method.
\end{abstract}

Keywords: Gradient Estimator, Nonpreemptive Priority Queues, Phantom Systems

\footnotetext{
* Supported in part by NSERC and FCAR Canadian grants. Work partially completed while the author was on leave at the Department of Electrical and Electronic Engineering, Melbourne University.

${ }^{\dagger}$ Supported in part by the Air Force Office of Scientific Research (F49620-98-1-0111) and the National Science Foundation (DMI-9907980).
} 


\section{Introduction}

Nonpreemptive priority queueing systems can be used to describe a wide variety of manufacturing, telecommunication, and service industry problems [8]. Such queueing systems can be modeled using discrete-event simulation. Simulation models of priority queues are useful for studying various steady-state responses (e.g., system sojourn times) associated with each customer class. The sensitivity of these response functions with respect to the input parameters allows one to assess how system outputs are affected by small changes to the inputs (e.g., customer arrival rates, service rates, and buffer space restrictions).

When the input parameters are continuous and the response functions are differentiable, sensitivity analysis is achieved from the appropriate gradients. Gradient estimators can be used as part of simulation optimization procedures, such as path search algorithms ([12], [15]). These algorithms iteratively determine a search direction and a step size to take in the chosen direction until some stopping criterion is met. For surveys of the simulation optimization literature, see [1], [4], [16], [20], or [21].

Over the past two decades, a great deal of research attention has been devoted towards the development of discrete-event simulation steady-state gradient estimators. The crude finite differences gradient estimator [3], though widely applicable, is highly inefficient [1]. Infinitesimal perturbation analysis and likelihood ratios/score functions [17] offer efficient alternatives, though their domains of applicability are more restricted than the finite differences estimator. Moreover, infinitesimal perturbation analysis is not applicable to priority queueing systems [5]. The harmonic gradient (HG) estimator [13] offers an approach that effectively simultaneously computes the finite differences estimator using multiple frequencies that are all close to zero, hence equivalently creating a parallel finite differences method. The phantom rare perturbation analysis (RPA) method [2] is similar to smoothed perturbation analysis [7], though it may require significant computational effort [25].

This paper introduces a new gradient estimator, termed the Phantom Harmonic Gradient (PHG) estimator. The phantom methodology introduces phantom arrivals into the queueing system and quantifies their effect on the response function. The PHG estimator exploits the parallelism offered by the harmonic gradient estimator, while using the phantom methodology to avoid perturbing the queueing system, hence eliminating some of the implementation problems associated with applying the harmonic gradient estimator directly. The paper is organized as follows. Section 2 presents notation and definitions that are needed to develop the PHG estimator. Section 3 summarizes the harmonic gradient estimator. Section 4 discusses phantom estimation and introduces the PHG estimator. The form of the PHG estimator allows both common and antithetic random numbers to be used simultaneously as a variance reduction technique. Section 5 looks 
at the variance of the PHG estimator. Section 6 presents computational results with the PHG estimator. These results suggest that the PHG estimator using the variance reduction technique provides an effective tool for estimating the gradient of the steady state response function. Section 7 summarizes the results presented.

\section{Model and Notation}

Consider a nonpreemptive priority queueing system with $C$ customer classes, where class $c_{i}$ customers have priority over class $c_{j}$ customers if $c_{i}<c_{j}$. Let $N_{c}(t)$, represent the (renewal) arrival process for class $c=1,2, \ldots, C$, where these arrival process are assumed to be independent. Assume that the queueing system operates under parallel admission control strategies, hence each arrival to the system may be thinned (i.e., accepted or rejected). Let the decisions of accepting customers of class $c$ be independent Bernoulli random variables with probability $p_{c}$. Let $\left\{\lambda_{c}, c=1,2, \ldots, C\right\}$ denote the effective arrival rates, after thinning, for each customer class. The service times for each customer class are assumed to be independent and identically distributed (IID) random variables with bounded second moments. In addition, assume that the queueing system is stable (i.e., the utilization factor is less than one).

The system response function for the queueing system, $F(\lambda)$, is a function of the average system time over either a finite or an infinite horizon, where the objective is to estimate the sensitivity of this function with respect to the effective arrival rates. Note that in the infinite horizon case, the limit measure exists and is unique, since the queue is assumed to be stable. If the arrival process is Poisson, then the derivative with respect to $\lambda_{c}$ is the same as the derivative with respect to $p_{c}$ evaluated at $p_{c}=1$. If the arrival process follows some other (e.g., general) renewal process, then the derivative with respect to the rate is not (in general) uniquely defined. Since the system is under admission control, then the relevant sensitivity is with respect to the admission control parameter. Therefore, $\frac{\partial}{\partial \lambda_{c}} F(\lambda)$ is defined as $\left.\frac{\partial}{\partial p_{c}} F(\lambda)\right\rfloor_{p_{c}=1}$.

There are several challenges associated with estimating the gradients for this system using a single simulation run. First, even if $\lambda_{c}$ is assumed to be a scale parameter of the interarrival times for class $c$, infinitesimal perturbation analysis (IPA) cannot be applied [5], since a Lipschitz continuity condition on the sample performance $h(\lambda, \omega)$ is required for the stochastic derivative to be unbiased (i.e., $\frac{\partial}{\partial \lambda_{c}} \mathrm{E}[h(\lambda)]=\mathrm{E}\left[\frac{\partial}{\partial \lambda_{c}} h^{\prime}(\lambda)\right]$ ). Priority service disciplines lead to a reordering of customers (i.e., customers do not depart in the same order in which they arrive), hence IPA is not applicable because infinitesimal changes of one arrival rate may cause discontinuities in the waiting times per class.

The likelihood ratio method can be applied to the Bernoulli decision variables, but cannot be used for evaluating the sensitivities at $p_{c}=1$. To see this, note that this method is based on the 
following result (see [22]): if a family of probability measures $\nu_{\theta}, \theta \in[0,1]$ is dominated by a measure $\mu$, where $f_{\theta}(x)$ denotes the $\mu$-density of $\nu_{\theta}$ and $S_{\theta}(x)=\frac{\partial}{\partial \theta} \ln f_{\theta}(x)$ denotes the "score function", then $\frac{\partial}{\partial \theta} \mathrm{E}_{\theta}[g(X)]=\mathrm{E}\left[g(X) S_{\theta}(X)\right]$ for any integrable function $g$, where the first expectation is with respect to $\nu_{\theta}$ and the second expectation is with respect to $\mu$. In the case of Bernoulli variables with parameter $\theta$ and $\mu=\nu_{\theta_{0}}$ for $0<\theta_{0}<1$, then for any integrable function $g(x)$,

$$
\mathrm{E}_{\theta}[g(X)]=g(0)(1-\theta)+g(1) \theta=g(0)\left(\frac{1-\theta}{1-\theta_{0}}\right)\left(1-\theta_{0}\right)+g(1)\left(\frac{\theta}{\theta_{0}}\right) \theta_{0}=\mathrm{E}\left[g(X) f_{\theta}(X)\right]
$$

where the $\mu$-density of $\nu_{\theta}$ is given by the random variable

$$
f_{\theta}(x)= \begin{cases}\left(\frac{1-\theta}{1-\theta_{0}}\right) & \text { if } x=0 \\ \left(\frac{\theta}{\theta_{0}}\right) & \text { if } x=1\end{cases}
$$

Therefore the score function is $S_{\theta}(x)=-(1-\theta)^{-1} \mathbf{1}_{\{x=0\}}+\theta^{-1} \mathbf{1}_{\{x=1\}}$, which is unbounded as either $\theta \rightarrow 0$ or $\theta \rightarrow 1$. This result reflects the fact that at the extreme values the measure in question is degenerate.

Smoothed perturbation analysis (SPA) can be applied to nonpreemptive priority queueing systems, though it is equivalent to the RPA method [2] and requires a significant computation effort [25]. A different SPA for this problem is proposed in [18], requiring a fifth bounded moment of the service distribution. The finite differences method and the HG estimator both require perturbing the input parameters of the system, which leads to added bias in the estimators when performed in a single simulation run [13], [9]. These perturbations, while straightforward to implement for simulation experiments, are undesirable for on-line adaptive control of systems that take measurements to construct the sensitivity estimators.

These observations suggest the need for an efficient approach to estimate gradients for nonpreemptive priority queueing system responses. The following notation and definitions are needed to develop an estimator to fill this void, termed the PHG estimator. The PHG estimator incorporates the phantom methodology within the HG estimator framework.

\section{Notation:}

$\mathbf{1}_{\{A\}}:$ the indicator function of event $A$

$T_{n} \quad$ : arrival time of the $n^{\text {th }}$ customer to arrive into the system

$Z_{n} \quad: \quad$ service time of the $n^{\text {th }}$ customer to arrive into the system

$\tau_{i}:$ arrival time of the $i^{\text {th }}$ customer to depart from the system

$n(i)=\sum_{j=1}^{N} I_{\left\{\tau_{j} \leq \tau_{i}\right\}}, i=1,2, \ldots, N$, the arrival number of the

$i^{\text {th }}$ customer departing from the system. Therefore, $\tau_{i} \equiv T_{n(i)}$.

$A_{i}=Z_{n(i)}$, the interarrival time between the $n(i)^{t h}$ and $n(i+1)^{t} h$ customers arriving to the system 
$S_{i}=Z_{n(i)}$, the service time of the $i^{\text {th }}$ customer to depart from the system

$I_{c}[n]=1(0)$ if the $n^{\text {th }}$ customer that arrives to the system is (not) of class $c$

$\lambda=\left(\lambda_{1}, \lambda_{2}, \ldots, \lambda_{C}\right)$

$\lambda_{0}=\sum_{c=1}^{C} \lambda_{c}$, the overall arrival rate

By definition, $A_{i}=T_{n(i+1)}-T_{n(i)}$. Moreover, $A_{i}$ represents the interarrival time between the $i^{\text {th }}$ and $(i+1)^{t h}$ customers departing the system. Note that if the $(i+1)^{t h}$ customer departing the system has higher priority than the $i^{\text {th }}$ customer departing the system, then $A_{i}$ may be negative.

Although customers do not depart in the same order in which they arrive, all customers that arrive within a busy period also depart within the same busy period (though not necessarily in the same order). Therefore, $n(i)=i$ if the $i^{\text {th }}$ customer that arrives to the system also is the first customer in a busy period.

Customers are labeled according to the order in which they depart from the system (this will be discussed more fully in Section 4$)$. Let $F_{c}(\lambda)$ denote the steady-state expected sojourn time for class $c$. The system response function is assumed to be a function of $F_{c}(\lambda)$. Define $X_{i}$ to be the sojourn time for the $i^{\text {th }}$ customer departing from the system. For a fixed number of customers $N$ (i.e., the total number of customers over all $C$ classes), the sample average

$$
\lim _{N \rightarrow \infty} \frac{\lambda_{0}}{\lambda_{c} N} \sum_{i=1}^{N} X_{i} I_{c}[n(i)]=F_{c}(\lambda) \text { with probability one (w.p.1) }
$$

is a (strongly) consistent estimator for $F_{c}(\lambda)$, since the ratio of the number of customers of class $c$ to the total number of customers $(N)$ approaches $\lambda_{c} / \lambda_{0}$ w.p.1 as $N$ approaches infinity (provided the queueing system is stable).

\section{The Harmonic Gradient Estimator}

The harmonic gradient estimator requires the input parameters to be (simultaneously) sinusoidally varied during a single simulation run. For a nonpreemptive priority queueing system, set

$$
\lambda_{c}(t)=\lambda_{c}(0)+a_{c} \sin \left(2 \pi \omega_{c} t\right)
$$

$t=0,1, \ldots, T, c=1,2, \ldots, C$, where $\lambda_{c}(t)$ is the arrival rate for class $c$ customers at index $t, \omega_{c}$ are the oscillation frequencies, $a_{c}$ are the oscillation amplitudes, and $T$ is the number of customers of each class that exit the system during one "period" of the simulation run. Let $X_{c, n(i)}(\lambda)=X_{i} I_{c}[n(i)]$ be a system response for the $n(i)^{t h}$ customer of class $c$ (e.g., the sojourn system time), where (1) holds. 
The oscillation frequencies are typically chosen to be Fourier frequencies (i.e., $\omega_{c}=h_{c} / T$ for $h_{c} \in\{1,2, \ldots,\lfloor T / 2\rfloor\}$ ) (see [9]). The harmonic gradient estimator is defined as

$$
\hat{A}\left(a_{c}, \omega_{c}\right)=\frac{2}{a_{c} T^{\prime}} \sum_{t=0}^{T^{\prime}} X_{c, t}(\lambda(t)) \sin \left(2 \pi \omega_{c} t\right),
$$

where $\lambda(t)=\left(\lambda_{1}(t), \lambda_{2}(t), \ldots, \lambda_{c}(t)\right)$ and $T^{\prime}=r T$ for some $r \in \mathbb{Z}^{+}$. If $F_{c}(\lambda)$ can be locally approximated by a second-order Taylor series expansion, then $\hat{A}\left(a_{c}, \omega_{c}\right) \rightarrow \partial F_{c}(\lambda) / \partial \lambda_{c}$ in probability, as $\left(\omega_{c}, a_{c}\right) \rightarrow(0,0)($ see $[13])$.

One difficulty associated with estimating multiple gradient components simultaneously is the associated frequency selection problem $[10,11]$. As the number of input parameters increase, the potential for frequency confounding (or overlapping) also increases. More specifically, higher order derivative terms can bias the gradient estimates, even if the oscillation frequencies are chosen to minimize this effect (see [13], [10] for discussions on the difficulties associated with the frequency selection problem). The PHG estimator introduced in Section 4 is formulated and designed to overcome this problem.

\section{The Phantom Harmonic Gradient}

\subsection{Phantom Estimation}

To describe a phantom system, several additional terms must be defined. A nominal system is defined by the input stream of arrivals for class $c, N_{c}(t)$, the service time distributions, and the service discipline. The nominal trajectory follows the dynamics of the queue, given the sequence of arrival and service times, $\left(T_{1}, Z_{1} ; T_{2}, Z_{2} ; \ldots\right)$. The input streams are assumed to be independent renewal processes, and $N_{c}(t) / t \rightarrow \bar{\lambda}_{c}$ w.p.1, as $t \rightarrow \infty$ ([14]). This system is assumed to be stable for the vector of input rates $\bar{\lambda}$. Therefore, in the nominal system, Lindley's equation is

$$
X_{i}=\left[X_{i-1}-A_{i-1}\right]^{+}+S_{i}
$$

where $[x]^{+}=\max \{x, 0\}$.

Define $\eta=\left\{\eta_{n}\right\}, n=1,2, \ldots$ to be a sequence of binary decision variables (where each such variable is associated with a particular customer). A phantom system is defined in the same way as a nominal system, except that it is coupled with a particular sequence of binary decision variables $\eta$ in the following way. If $\eta_{i}=1$ (or $\eta_{i}=0$ ), then the associated customer $i$ is (or is not) allowed to enter the system. An $\eta$-phantom trajectory follows the dynamics of the queue, given the sequence of arrival and service times, $\left(T_{1}, Z_{1} \eta_{1} ; T_{2}, Z_{2} \eta_{2} ; \ldots\right)$. Therefore, for a phantom system with binary decision variables $\eta$, the arrival time for the $n^{\text {th }}$ customer is $T_{n}$ (as for the nominal system), with 
service time $Z_{n} \eta_{n}$. A customer $i$ with $\eta_{i}=0$ is a phantom customer with service time zero, hence this customer vanishes from the system at the instant at which it is scheduled to begin service.

The effective arrival rates for the $\eta$-phantom system are $\lambda_{c}(\eta)=\bar{\lambda}_{c} q_{c}, c=1,2, \ldots, C$, where $q_{c}$ is the fraction of customers of class $c$ allowed entry into the phantom system,

$$
q_{c}=\lim _{N \rightarrow \infty} \frac{\sum_{n=1}^{N} \eta_{n} I_{c}[n]}{\sum_{n=1}^{N} I_{c}[n]} \quad \text { w.p.1. . }
$$

The order in which customers complete service may be different for the nominal and the phantom systems, since some of the customers in the nominal system are no longer served in the phantom system. However, the order of arrivals and the arrival epochs for the nominal and phantom systems are identical. Consequently, let $n(\eta, i)$ be the arrival number of the $i^{\text {th }}$ customer departing the $\eta$ phantom system. Since the interarrival times also depend on $\eta$, then $A_{i}(\eta)=T_{n(\eta, i+1)}-T_{n(\eta, i)}$ is the interarrival time between the $i^{\text {th }}$ and $i+1^{\text {th }}$ departures from the $\eta$-phantom system, and the service time of the $i^{\text {th }}$ departing customer in this system is $S_{i}(\eta)=Z_{n(\eta, i)} \eta_{n(\eta, i)}$. Therefore, Lindley's equation (now termed the modified Lindley equation) becomes

$$
X_{i}(\eta)=\left[X_{i-1}(\eta)-A_{i-1}(\eta)\right]^{+}+S_{i}(\eta)
$$

where $X_{i}(\eta)$ is the sojourn time for the $i^{\text {th }}$ customer departing the queue, provided $\eta_{n(\eta, i)}=1$. Otherwise, (3) keeps track of the waiting times and arrival times so that it remains valid for future customers [24]. Note that $X_{i}(\underline{1})$ denotes the sojourn times for the $i^{\text {th }}$ customer departing the nominal system (i.e., $\eta=\underline{1}=(1,1, \ldots))$.

The recursion in (3) is straightforward to evaluate, since each customer is assigned a service time and an arrival time, and the new order of customer service can be determined directly from the customers' classes and the arrival times. If a busy period has been completed with $\alpha$ customers in the nominal system, then this busy period must also have been completed in the phantom system. This follows from the observation that the time at which the last service of the first busy period occurs in the nominal system is $B_{1}=\sum_{i=1}^{\alpha} S_{i}$, with $T_{\alpha+1}>B_{1}$. Since the server serves consecutive customers during a busy period, then the actual order in which service takes place does not affect $B_{1}$. Service times in the phantom system either are the nominal service times or zero. Therefore for any binary decision sequence $\eta$ the service completion epoch of the first busy period of the $\eta$-phantom system satisfies

$$
B_{1}(\eta) \leq \sum_{i=1}^{\alpha} S_{i}(\eta)=\sum_{n=1}^{\alpha} Z_{n} \eta_{n}<B_{1}(\underline{1})<T_{\alpha+1},
$$

hence customer $\alpha+1$ must also begin a busy period in the phantom system. This stochastic monotonicity property is referred to as stochastic domination of the phantom systems by the nominal system. Lastly, if the $i^{\text {th }}$ customer that departs the nominal system begins a busy period in the nominal system, then $n(i)=n(\eta, i)=i$ for all decision sequences $\eta$. 


\subsection{PHG Gradient Estimation}

This section formally introduces the Phantom Harmonic Gradient (PHG) estimator. The gradient estimator is constructed by simultaneously computing each derivative component. To simplify the description and analysis of the PHG estimator, only the PHG derivative estimator with respect to a single parameter, $\lambda_{1}$ is presented; the PHG estimator for the other parameters is obtained in an analogous way.

Assumption 1 is used to define the PHG estimator.

Assumption 1 Suppose that for a stable nonpreemptive priority queueing system with arrival rates $\lambda$, there exists a real number $a>0$ such that the queueing system with arrival rates $\lambda(1+a)$ (i.e., a scaling up of the arrival rates by a factor of $1+$ a) is also stable.

To obtain an expression for the PHG derivative estimator with respect to $\lambda_{1}$, the nominal system is built with the arrival rates

$$
\bar{\lambda}_{c}=\lambda_{c}(1+a), \quad c=1,2, \ldots, C, \quad \bar{\lambda}_{0}=\sum_{c=1}^{C} \bar{\lambda}_{c} .
$$

Let $T$ be an integer greater than or equal to three, $\omega=1 / T$, and

$$
p_{t}(c)=\frac{1+a \sin (2 \pi \omega t) \mathbf{1}_{\{c=1\}}}{1+a}= \begin{cases}\frac{1+a \sin (2 \pi \omega t)}{1+a} & \text { if } c=1 \\ \frac{1}{1+a} & \text { otherwise }\end{cases}
$$

for $t=0,1, \ldots, T-1$. Also, let $\eta(t), t=0,1, \ldots, T-1$, denote a sequence of independent Bernoulli random variable vectors with

$$
\eta_{i}(t) \sim \operatorname{Bernoulli}\left(\theta_{i}(t)\right), \quad \text { where } \theta_{i}(t)=\sum_{c=1}^{C} p_{t}(c) I_{c}[n(i)],
$$

such that $\theta_{i}(t)=p_{t}(c)$ if the $i$-th customer to leave the phantom queue is of class $c$. This is equivalent to assigning a $\operatorname{Bernoulli}\left(p_{t}(c)\right)$ variable to each arriving customer of class $c$, hence the $\eta(t)$-phantom system follows the dynamics of a nonpreemptive priority queue with effective rates

$$
\lambda_{1}(t)=\lambda_{1}(1+a \sin (2 \pi \omega t)), \quad \lambda_{c}(t)=\lambda_{c}, c=2,3, \ldots, C
$$

Remark: The case with $t=0$ corresponds to what is referred to as the base system, which has arrival rates $\lambda_{c}, c=1,2, \ldots, C$. This is the system for which the derivatives are to be estimated, although (as will be seen from the results that follow) the base system does not need to be simulated to perform the gradient estimation.

From a single simulation run of the nominal system with arrival rates $\bar{\lambda}$, the $T-1$ phantom systems can all be observed simultaneously with common random variables for the interarrival and 
service times. The nominal arrival rate is the maximum value of the sinusoidal function, where other rates are thinned out to correspond to the chosen phantom rates. Finally, to simplify the notation, let $n_{t}(i)$ denote $n(\eta(t), i)$ for the arrival number of the $i^{\text {th }}$ departing customer from the phantom system labelled by $t$.

The following theorem shows that the phantom harmonic gradient (PHG) estimator is strongly consistent and strongly consistent in the average sense.

Theorem 1 Let $N \in \mathbb{Z}^{+}$be a deterministic number of service completions in the nominal system. For $m \in \mathbb{Z}^{+}$, define the statistic

$$
\hat{X}_{N, m}^{c}(t)=\frac{\bar{\lambda}_{0}}{\lambda_{c}(t) N} \sum_{i=m N+1}^{m(N+1)} X_{i}(\eta(t)) \eta_{i}(t) I_{c}\left[n_{t}(i)\right]
$$

and the phantom harmonic gradient $(P H G)$ estimator for $\partial F_{c}(\lambda) / \partial \lambda_{1}$

$$
\hat{D}_{1}^{c}(N, n)=\frac{1}{a \lambda_{1}(0) T} \sum_{t=1}^{T-1} \hat{X}_{N, n}^{c}(t) \sin (2 \pi \omega t) .
$$

Then for all $m \in \mathbb{Z}^{+}$,

$$
\lim _{N \rightarrow \infty} \hat{D}_{1}^{c}(N, m)=\frac{\partial}{\partial \lambda_{1}} F_{c}(\lambda)+\mathcal{O}\left(a^{2}\right) \quad \text { w.p.1 }
$$

and for all $N \in \mathbb{Z}^{+}$,

$$
\lim _{M \rightarrow \infty} \frac{1}{M} \sum_{m=0}^{M-1} \hat{D}_{1}^{c}(N, m)=\frac{\partial}{\partial \lambda_{1}} F_{c}(\lambda)+\mathcal{O}\left(a^{2}\right) \quad \text { w.p.1 }
$$

Remark: Strong consistency (7) in Theorem 1 is needed for strong convergence in simulation optimization, when the derivative estimators require an increasing estimation interval length $N$, while the second property (8), referred to in [21] and [23] as "strong consistency in the average sense," yields convergence of on-line optimization algorithms, where $N$ is a constant (see [15] and [23] for details).

Proof : First, as in (1), it is necessary to show that for each value of $t=1,2, \ldots, T-1$,

$$
\hat{X}_{N, m}^{c}(t) \rightarrow F_{c}[\lambda(t)] \quad \text { w.p.1 as } N \rightarrow \infty \text {. }
$$

Let $N_{c}(m, N)$ denote the number of class $c$ customers that have been served from among the $N$ customers within departing customers $m N+1$ and $m N+N$ in the phantom system $t$. Then by construction,

$$
\frac{N_{c}(m, N)}{N} \rightarrow \frac{\lambda_{c}(t)}{\bar{\lambda}_{0}} \quad \text { w.p.1 as } N \rightarrow \infty
$$


given the stability assumption (Assumption 1). On the other hand, by the strong law of large numbers,

$$
\lim _{N \rightarrow \infty} \frac{1}{N_{c}(m, N)} \sum_{i=m N+1}^{m(N+1)} X_{i}(\eta(t)) \eta_{i}(t) I_{c}\left[n_{t}(i)\right]=F_{c}(t) \quad \text { w.p.1, }
$$

where $F_{c}(t)$ is assumed to be bounded for all $t$. By the Continuous Mapping Theorem, these two results establish (9). To show (7), first note that the phantom systems components in (6) are summed. Using (9),

$$
\hat{D}_{1}^{c}(N, m) \rightarrow \frac{1}{a \lambda_{1}(0) T} \sum_{t=1}^{T-1} F_{c}[\lambda(t)] \sin (2 \pi \omega t) \quad \text { w.p.1 as } N \rightarrow \infty .
$$

The result in (7) is then obtained using the Fourier decomposition of $F_{c}(\lambda(t))$, which is assumed to be continuously differentiable. To simplify the notation, fix all the other components of the vector $\lambda$ at $\lambda_{c}(0), c \neq 1$, and write $F_{c}\left(\lambda_{1}\right)$ as a function of one variable. Then $G(t) \equiv F_{c}\left(\lambda_{1}(0)+\delta(t)\right)$ is a smooth, periodic, analytical function on $t \in[0,1)$, with

$$
\delta(t)=a_{1} \sin (2 \pi t), \quad a_{1}=a \lambda_{1}(0) .
$$

Therefore, $G(t)$ has the Fourier representation [19]:

$$
G(t)=G(0)+\sum_{k=1}^{\infty} g_{k} \sin (k \pi t), \quad \text { with } g_{k}=2 \int_{0}^{1} G(t) \sin (k \pi t) d t .
$$

Taking derivatives, $G^{\prime}(t)=G(0)+\sum_{k=1}^{\infty}\left(k \pi g_{k}\right) \cos (k \pi t)$, which implies that $g_{k}$ can also be represented as

$$
g_{k}=\frac{2}{k \pi} \int_{0}^{1} G^{\prime}(t) \cos (k \pi t) d t .
$$

Applying the chain rule, $G^{\prime}(t)=F_{c}^{\prime}\left[\lambda_{1}(0)+\delta(t)\right] \delta^{\prime}(t)$, where $\delta^{\prime}(t)=2 \pi a_{1} \cos (2 \pi t)$. Moreover, using a Taylor series expansion for $F_{c}^{\prime}\left[\lambda_{1}(0)+\delta(t)\right]$ for small values of $a($ and $\delta(t))$,

$$
G^{\prime}(t)=2 \pi a_{1} \cos (2 \pi t)\left(\frac{\partial F_{c}}{\partial \lambda_{1}}\left[\lambda_{1}(0)\right]+\delta(t) \frac{\partial F_{c}^{2}}{\partial \lambda_{1}^{2}}\left[\lambda_{1}(0)\right]\right)+\mathcal{O}\left(a_{1}^{3}\right) .
$$

Substituting this expression into (12) (with $k=2$ ) leads to

$$
g_{2}=a_{1} \frac{\partial F_{c}}{\partial \lambda_{1}}\left[\lambda_{1}(0)\right]+\mathcal{O}\left(a_{1}^{3}\right)
$$

which follows from the orthonormality of the trigonometric functions as well as the identities

$$
\begin{aligned}
& 2 \int_{0}^{1} \cos ^{2}(k \pi t) d t=1, \text { for any integer } k \geq 1 \\
& \int_{0}^{1} \sin (2 \pi t) \cos ^{2}(2 \pi t) d t=0 .
\end{aligned}
$$

Lastly, setting the last expression for $g_{2}$ equal to (11) (with $k=2$ ) leads to

$$
\frac{\partial F_{c}}{\partial \lambda_{1}}\left[\lambda_{1}(0)\right]=\frac{2}{a_{1}} \int_{0}^{1} G(t) \sin (2 \pi t) d t+\mathcal{O}\left(a_{1}^{2}\right) .
$$


If $F_{c}\left(\lambda_{1}\right)$ is a polynomial function of order less than $T \in \mathbb{Z}^{+}$, then this integral is a discrete average over equally spaced points [26] and

$$
\int_{0}^{1} F_{c}\left(\lambda_{1}+a_{1} \sin (2 \pi t)\right) \sin (2 \pi t) d t=\frac{1}{T} \sum_{t=0}^{T-1} F_{c}\left(\lambda_{1}+a_{1} \sin (2 \pi \omega t)\right) \sin (2 \pi \omega t)
$$

with $\omega=1 / T$, which establishes (7). Moreover, if $F_{c}$ is analytical, then it can be approximated using a Taylor series expansion, and the left and right hand side of (14) can be made arbitrarily close, as $T$ approaches infinity [26].

To establish (8), note that $\hat{D}_{1}^{c}(N, m)$ is an additive estimator, which implies that

$$
\frac{1}{M} \sum_{m=0}^{M-1} \hat{D}_{1}^{c}(N, m)=\hat{D}_{1}^{c}(M N, 0)
$$

and the result follows.

The nominal system dominates all the phantoms, hence by stochastic monotonicity, at the beginning of each nominal busy period all the phantom systems are also empty. Therefore stability of the nominal system implies stability of the phantoms and the variance of $\hat{D}_{1}^{c}(N, m)$ decreases as $\mathcal{O}(1 / N)$ for any $m \in \mathbb{Z}^{+}$.

The form of the PHG estimator eliminates the bias problems associated with the HG estimators, since the sinusoidal perturbations are now performed simultaneously or in parallel (across the sinusoidally varied input parameter values). In particular, the oscillation frequencies no longer must approach zero, since the sample mean estimators are consistent for the stationary averages. Moreover, to estimate the gradient, the same oscillation frequency can be used for each input parameter $\lambda_{c}, c=1,2, \ldots, C$. Note that from (6), computations for $T-1$ parallel systems are needed for each derivative component, hence a total of $C(T-1)$ parallel phantom systems are needed to compute all $C$ gradient components.

In practice, the required phantom systems can be obtained from the nominal simulation by generating Bernoulli $\left(p_{t}(c)\right)$ decision sequences for each $t=1, \ldots, T-1$. The modified Lindley equations (3) can be used to evaluate the PHG estimator by generating only the decisions $\eta_{n}(t), t=$ $1, \ldots, T-1$ for each incoming customer. As stated in Theorem 1, if one wants to estimate the derivative of the base system at arrival rate $\lambda$, then one has to simulate a system at the nominal rate $\bar{\lambda}$. However, if on-line estimation is sought, then the PHG estimator yields an estimate for a base system with slightly different parameters than the observed system.

\section{Variance of the PHG Estimator}

This section analyzes the variance of the PHG estimator by presenting a simple upper bound expression that is used to establish two results. The first result is that the expectation of the 
conditional variance of the PHG estimator tends to zero as $T \rightarrow \infty$. The second result is that this upper bound can be used to assess the rate at which the variance of the PHG estimator approaches zero. A variance reduction technique that simultaneously uses both common and antithetic random numbers is also presented.

\subsection{An Upper Bound for the Variance of the PHG Estimator}

Let $T=2 M+1$, with $M \geq 1$ a positive integer. The upper bound expression for the variance of the PHG estimator in (6) is obtained for the derivative of the steady state response function for class $c$ with respect to $\lambda_{1}$. From (5), define

$$
W\left(\lambda_{1}+\delta(t)\right) \equiv \hat{X}_{N, 0}^{c}(t), \quad t=0,1, \ldots, T-1
$$

where $\delta(t)=a_{1} \sin (2 \pi \omega t), \omega=1 / T$ and $a_{1} \equiv a \lambda_{1}(0)$, as defined in the proof of Theorem 1 in Section 4. From the Markovian structure of Lindley's equation (3), the initial state does not influence the asymptotic distribution of $\mathrm{W}$ as $N$ grows, provided that the nominal system is stable. Without loss of generality, assume that the initial state at $t=0$ is an empty system.

A nominal system is simulated (or observed) with arrival rates $\lambda_{c}+a_{c}, a_{c}=a \lambda_{c}$, until $N$ service completions occur, where $N \in \mathbb{Z}^{+}$is deterministic. As the customers arrive to the nominal system, uniform random variates are used to generate the $T$ Bernoulli sequences $\eta_{i}(t) ; t=1, \ldots, T-1$ that define the phantom systems. From the modified Lindley's equation (3), the $T-1$ statistics $W\left(\lambda_{1}+\delta(t)\right), t=1, \ldots, T-1$ are computed simultaneously.

Consider the conditional expectation and variance of the PHG estimator given the nominal system trajectory $\xi=\left\{\left(T_{n}, Z_{n}\right), n=1, \ldots, \tau(N)\right\}$, where $\tau(N)$ denotes the customer number corresponding to the index of the last customer to arrive in the nominal queue before the $N^{\text {th }}$ service completion. The unconditional variance can be obtained using the equation

$$
\operatorname{Var}\left[D_{1}^{c}(N, 0)\right]=\operatorname{Var}\left[\mathrm{E}\left[D_{1}^{c}(N, 0) \mid \xi\right]\right]+\mathrm{E}\left[\operatorname{Var}\left[D_{1}^{c}(N, 0) \mid \xi\right]\right]
$$

Given the nominal system trajectory, $\xi$, the PHG estimator is a function of the random decision sequences. Moreover, the conditional expectation and variance of the PHG estimator are functions of $\xi$, hence are random variables. Using the notation $W(\cdot \mid \xi)$ to denote the random variable $\mathrm{W}$ given the nominal trajectory $\xi$, then from Theorem 1, $\operatorname{Var}\left[\mathrm{E}\left[D_{1}^{c}(N, 0) \mid \xi\right]\right]=O(1 / N)$ for $N$ large. Moreover, the rate at which the unconditional variance, $\operatorname{Var}\left[D_{1}^{c}(N, 0)\right]$, approaches zero is the slower of $\mathcal{O}(1 / N)$ and the rate at which $E\left[\operatorname{Var}\left[D_{1}^{c}(N, 0) \mid \xi\right]\right]$ approaches zero (for $T$ large).

To assess the variance function of the PHG estimator, the conditional variance of the PHG estimator is analyzed as a function of $T$, for fixed $N$. In Lemma 1 and Theorem 2, whenever the term "w.p.1" is used for conditional expectations, conditional variances or conditional covariances, 
this is with respect to the underlying probability measure that governs $\xi$, the trajectory of the nominal system.

Lemma 1 Under Assumption 1, if all the conditional gradient and Hessian components of $W(\lambda \mid \xi)$ are finite w.p.1, then

$$
v(N, \xi) \equiv \max _{0 \leq t \leq T-1}\left\{\operatorname{Var}\left[W\left(\lambda_{1}+\delta(t)\right)-W\left(\lambda_{1}-\delta(t)\right) \mid \xi\right]\right\}<\infty \text { w.p.1. }
$$

Moreover, $\mathrm{E}[v(N, \xi)]=\mathcal{O}\left(a_{1}^{2}\right)$.

Proof : Given Assumption 1, then $v(N \mid \xi)$ is finite w.p.1. Moreover, the second order Taylor series expansion of $\mathrm{E}[W(\lambda \mid \xi)]$ results in $\mathrm{E}[v(N \mid \xi)]=\mathcal{O}\left(a_{1}^{2}\right)$ w.p.1.

The results in Lemma 1 are independent of the correlation structure chosen to generate the Bernoulli sequences $\left\{\eta_{i}(t)\right\}$ for different $t=1,2, \ldots, T-1$.

Since $T=2 M+1$, then (6) can be rewritten as

$$
D_{1}^{c}(N, 0)=\frac{4}{T} \sum_{m=1}^{M} D(m) \sin ^{2}(2 \pi \omega m)
$$

where the finite differences

$$
D(m)=\frac{W\left(\lambda_{1}+\delta(m)\right)-W\left(\lambda_{1}-\delta(m)\right)}{2 \delta(m)}
$$

are well-defined (since for $m=1,2, \ldots, M, \sin (2 \pi \omega m)>0)$.

Expanding the conditional variance (for each trajectory $\xi$ ) of the summation in (16) leads to

$$
\begin{aligned}
& \operatorname{Var}\left[D_{1}^{c}(N, 0) \mid \xi\right]=\frac{16}{T^{2}}\left[\sum_{m=1}^{M} \operatorname{Var}\{D(m) \mid \xi\} \sin ^{4}(2 \pi \omega m)\right. \\
& \left.\quad+2 \sum_{m=1}^{M-1} \sum_{j=m+1}^{M} \operatorname{Cov}\{D(m), D(j) \mid \xi\} \sin ^{2}(2 \pi \omega m) \sin ^{2}(2 \pi \omega j)\right] .
\end{aligned}
$$

Since $\operatorname{Cov}\{D(m), D(j) \mid \xi\} \leq[\operatorname{Var}[D(m) \mid \xi]]^{1 / 2}[\operatorname{Var}[D(j) \mid \xi]]^{1 / 2}$ w.p.1 for all $m, j=1,2, \ldots, M$, then from Lemma 1, for each trajectory $\xi$,

$$
\operatorname{Cov}\{D(m), D(j) \mid \xi\} \leq \frac{v(N, \xi)}{4 a_{1}^{2} \sin (2 \pi \omega j) \sin (2 \pi \omega m)} \text { for all } m, j=1,2, \ldots, M,
$$

Substituting (19) into (18) leads to the crude upper bound for the conditional variance of the PHG estimator

$$
\operatorname{Var}\left[D_{1}^{c}(N, 0) \mid \xi\right] \leq \frac{4 v(N, \xi)}{a_{1}^{2} T^{2}}\left(\sum_{h=1}^{M} \sin (2 \pi \omega h)\right)^{2} \text { w.p.1. }
$$

Therefore, for $N$ (the length of the nominal system trajectory sample path simulation run) fixed, the conditional variance of the PHG estimator is bounded above by (20), where the upper 
bound depends on the number of phantom systems, $T=2 M+1$, for a given nominal system sample path trajectory $\xi$. For example, if $T=3$, then (20) equals $v(N, \xi) / 3 a_{1}^{2}$. This upper bound is monotonically increasing in $M$, and for each $\xi$,

$$
\begin{aligned}
\frac{4 v(N, \xi)}{a_{1}^{2}(2 M+1)^{2}}\left(\sum_{h=1}^{M} \sin (2 \pi \omega h)\right)^{2} & \rightarrow \frac{v(N, \xi)}{a_{1}^{2}}\left(\frac{1}{\pi} \int_{0}^{\pi} \sin (x) d x\right)^{2} \\
& =\left(\frac{2}{\pi a_{1}}\right)^{2} v(N, \xi),
\end{aligned}
$$

where the limit has bounded expectation (using Lemma 1). Therefore, by the Dominated Convergence Theorem, $\mathrm{E}\left[\operatorname{Var}\left(D_{1}^{c}(N, 0) \mid \xi\right)\right]$ is bounded as $T \rightarrow \infty$. This crude upper bound holds for any approach used to generate the phantom systems, i.e., no restrictions are placed on the correlation structure of the $T$ Bernoulli sequences $\left\{\eta_{i}(t)\right\}$ used to generate the phantom systems.

Theorem 2 shows that if the Bernoulli sequences (that generate the phantom systems) are independent, then the expectation of the conditional variance, $\operatorname{Var}\left[D_{1}^{c}(N, 0) \mid \xi\right]$ is $\mathcal{O}(1 / T)$ for $T$ large.

Theorem 2 Under Assumption 1, suppose that all the conditional gradient and Hessian components of $W(\lambda \mid \xi)$ are finite w.p.1.. If for $t \neq t^{\prime} \in\{1, \ldots, T-1\}$ the Bernoulli sequences $\eta(t)$ and $\eta\left(t^{\prime}\right)$ are independent, then $\mathrm{E}\left[\operatorname{Var}\left[D_{1}^{c}(N, 0) \mid \xi\right]\right]=O(1 / T)$ as $T \rightarrow \infty$.

Proof : If the Bernoulli sequences are independent across the indices $t$, then the conditional covariances (given the nominal system trajectory sample path $\xi$, hence the arrival epochs and service times) are zero. Therefore, the conditional variance expression in (18) is bounded above by

$$
\operatorname{Var}\left[D_{1}^{c}(N, 0) \mid \xi\right]=\frac{16}{T^{2}} \sum_{m=1}^{M} \frac{v(N, \xi) \sin ^{2}(2 \pi \omega m)}{4 a_{1}^{2}} \leq \frac{4 v(N, \xi)}{a_{1}^{2} T} \text { w.p.1. }
$$

From Lemma 1, by the Dominated Convergence Theorem, the expectation of $((21)$ is bounded above by a function that is $\mathcal{O}(1 / T)$, which establishes the result.

The result in Theorem 2, together with $\operatorname{Var}\left[\mathrm{E}\left[D_{1}^{c}(N, 0) \mid \xi\right]\right]=O(1 / N)$ for $N$ large ensures that from ((15)), a variance reduction effect is realized as both $T$ and $N$ increase. However, larger values for $T$ and $N$ result in an additional computational burden. The efficiency of an estimator must take into account the trade-off between precision and computational effort. Therefore, it would be preferable to identify ways to reduce the variance of the PHG estimator without increasing $T$ or $N$. The next section presents such a variance reduction technique.

\subsection{Variance Reduction for PHG Estimation}

The structure of the PHG estimator suggests that both common random numbers (CRN) and antithetic random numbers (ARN) can be used to reduce its variance. The PHG estimator is 
computed from a single nominal system simulation run of length $N$. The PHG estimation procedure simultaneously computes the gradient components by simultaneously generating all the phantom decision sequences. However, to simplify the description of the variance reduction technique, only the derivatives w.r.t $\lambda_{1}$ are considered.

Phantoms are created such that $W\left(\lambda_{1}+a_{1} \sin (2 \pi \omega t)\right), t=1,2, \ldots, T-1$, can be simultaneously computed from the nominal trajectory. The variance in (18) may be reduced by simultaneously using both CRN and ARN to generate the phantoms. To see this, divide the interval $(0, T)$ into four regions, as depicted in Figure 1 , with $K \in \mathbb{Z}^{+}$points in each region.

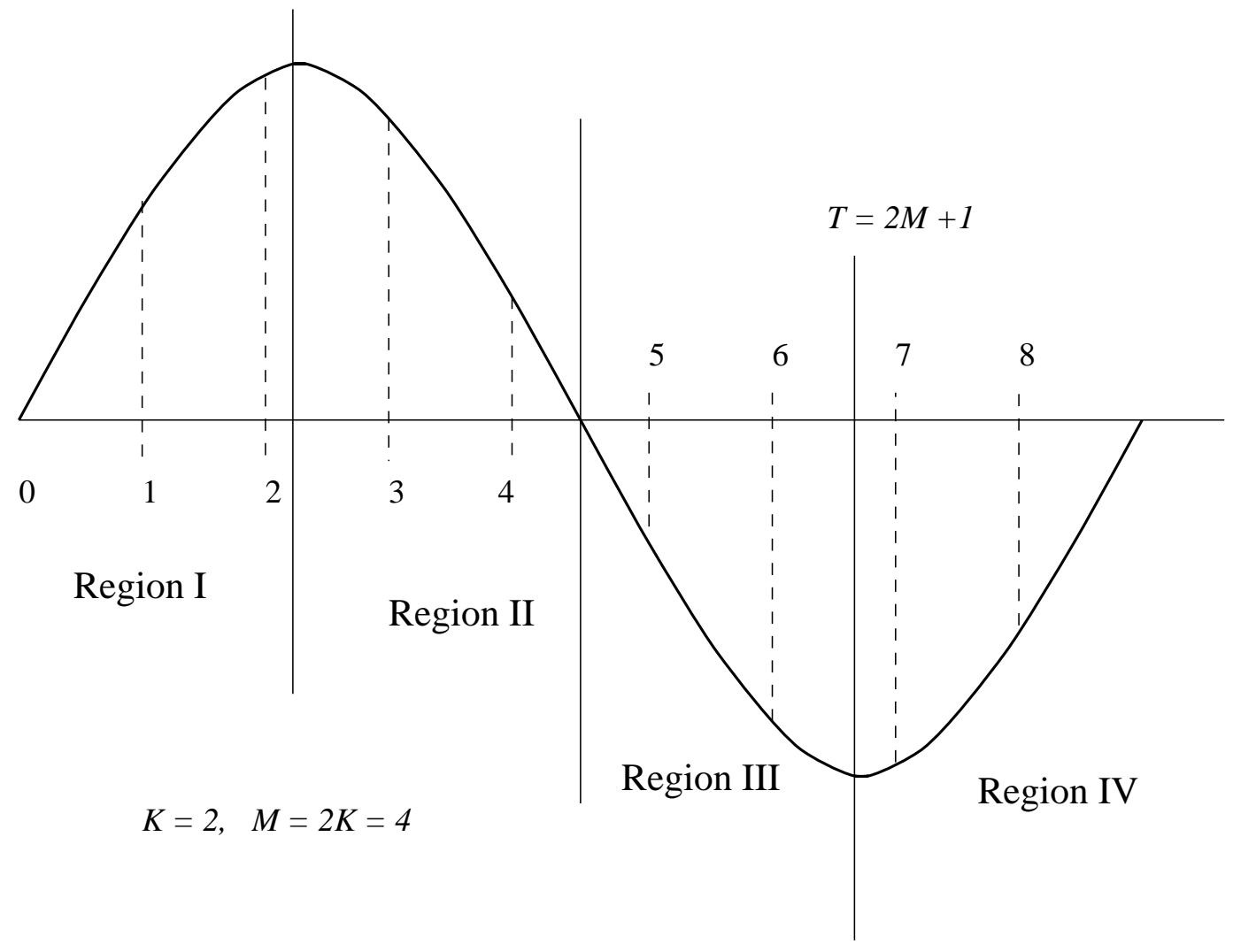

Figure 1: The Four Regions of $[0, T)$

Recall from (4) that for phantom system $t=1,2, \ldots, T-1$,

$$
p_{t}(c)=\frac{1+a \sin (2 \pi \omega t) I_{\{c=1\}}}{1+a} .
$$

When a customer $n$ arrives into the nominal system, and this customer is from class $c$, then $K$ IID $U(0,1)$ random variables $\left\{u_{n}(k), k=1,2, \ldots, K\right\}$ are used to define the phantom systems. Let $M=2 K$ and $T=2 M+1=4 K+1$. Then for each $k=1, \ldots, K$, define

$$
\begin{array}{rll}
\eta_{n}(k) & =I_{1}(n) I_{\left\{u_{n}(k)<p_{k}(1)\right\}} \quad(\text { Region I) } \\
\eta_{n}(T-k) & =I_{1}(n) I_{\left\{u_{n}(k)<p_{T-k}(1)\right\}} \quad(\text { Region IV) }
\end{array}
$$




$$
\begin{aligned}
\eta_{n}(M+1-k) & =I_{1}(n) I_{\left\{1-u_{n}(k)<p_{M+1-k}(1)\right\}} \quad \text { (Region II) } \\
\eta_{n}(M+k) & =I_{1}(n) I_{\left\{1-u_{n}(k)<p_{M+k}(1)\right\}} \quad(\text { Region III) }
\end{aligned}
$$

If the arriving customer $n$ is not of class 1 , then $p_{t}(c)=1 /(1+a)$ is independent of $t$ and only one random variate $u_{n} \sim U(0,1)$ is used, where $\eta_{n}(t)=I_{\left\{u_{n}<p_{t}(c)\right\}}$ for all $t$, with the corresponding arrival rate just the arrival rate of the base system (which is also the arrival rate at which the derivative is to be computed). Example 1 illustrates how these four regions are defined.

Example 1: Suppose that $K=3$, hence $T=13$. Therefore, there are 12 phantom systems. Figure 2 depicts the arrival rates for these 12 phantom systems. First, IID $U(0,1)$ variates are used to generate the three phantom systems 1, 2, and 3 (see (22), defined as Region I). To obtain the effect of CRN, this same set of $U(0,1)$ values are used to generate phantom systems 10,11 , and 12 (see (23), defined as Region IV). To obtain the effect of ARN, the same, though antithetic, set of IID $U(0,1)$ values are used to generate phantom systems 4,5 , and 6 (see $(24)$, defined as Region II) and phantom systems 7, 8, and 9 (see (25), defined as Region III). More specifically, phantom systems 1, 2, and 3 are matched with phantom systems 12, 11, and 10, respectively, using CRN, while phantom systems 4,5 , and 6 are matched with phantom systems 9,8 , and 7 , respectively, also using CRN. Moreover, phantom systems 1 and 12, 2 and 11, and 3 and 10, are matched with phantom systems 6 and 7, 5 and 8, and 4 and 9, respectively, using ARN. Lastly, the phantom systems in Regions I and IV are paired using CRN. Similarly, the phantom systems in Regions II and III are paired using CRN, which are antithetic to the random variates used in Regions I and IV. The CRN effect is analogous to using CRN to reduce the variance of the symmetric finite differences gradient estimator. The ARN effect, however, cannot be duplicated for the symmetric finite differences gradient estimator, hence provides here a means of further reducing the variance of the PHG estimator.

\section{Computational Results}

This section presents computational results with the PHG estimator and the variance reduction techniques. To obtain analytical results that can be compared to the simulation results and the PHG estimators introduced here, the simulation experiments were run for priority queueing systems with $C=2$, Poisson arrival processes, and exponential service time distributions. The arrival and service rates for a priority $c$ customer are given by $\lambda_{c}$ and $\mu_{c}$, respectively, $c=1,2$, where for the first set of experiments (i.e., system 1), $\mu \equiv \mu_{1}=\mu_{2}=1.0, \lambda_{1}=.45$, and $\lambda_{2}=.15$, while for the second set of experiments (i.e., system 2 ), $\mu \equiv \mu_{1}=\mu_{2}=1.0, \lambda_{1}=.10$, and $\lambda_{2}=.15$. The steady-state expected sojourn time for class $c$ customers is denoted by $F_{c}(\lambda)$, where $\lambda=\left(\lambda_{1}, \lambda_{2}\right)$. Each nominal system sample path simulation run was executed for $N=3000$ customers. All the 


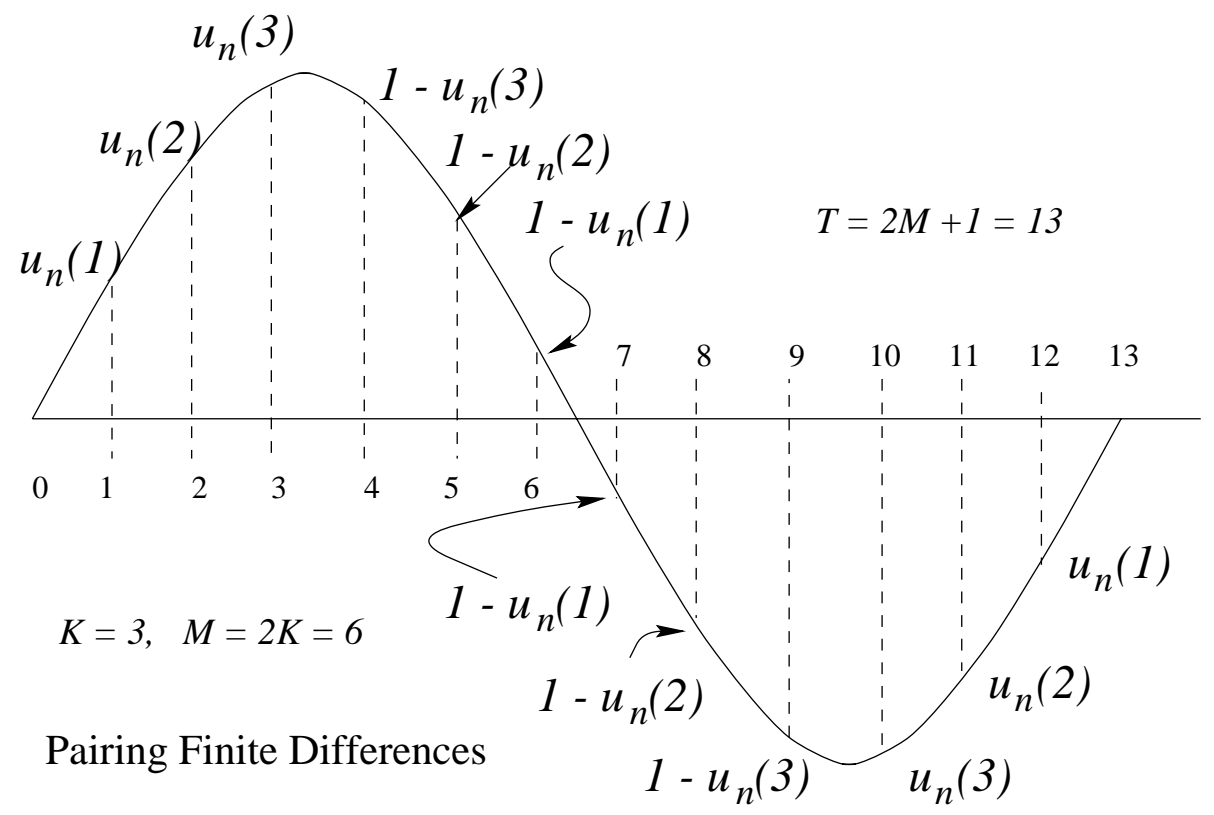

Figure 2: Antithetic and Common Random Numbers

experiments were run on the same workstation, hence the CPU times listed provide a means to compare the relative efficiency of the different estimators and variance reduction techniques. The analytical expressions for the steady state expected sojourn times for the two customer priorities are

$$
F_{1}(\lambda)=\frac{\lambda_{2}+\mu}{\mu\left(\mu-\lambda_{1}\right)}, \quad F_{2}(\lambda)=\frac{\lambda_{0}}{\left(\mu-\lambda_{1}\right)\left(\mu-\lambda_{0}\right)}+\frac{1}{\mu}
$$

where $\lambda_{0}=\lambda_{1}+\lambda_{2}$. The root mean squared error

$$
R M S E(Y)=\sqrt{\operatorname{Var}(Y)+[\mathrm{E}(Y-\theta)]^{2}}
$$

is used to measure how close the estimated gradient components are to their associated analytical values, where $Y$ denotes the estimator and $\theta$ denotes the output parameter that $Y$ is estimating. In addition, the total root mean squared error (TRMSE), the square root of the sum of the individual mean squared errors for the four gradient components obtained from each set of experiments, are be used to compare the different PHG estimators.

An amplitude of $1 \%$ of the input parameter value were used in the simulation runs to estimate the gradients (i.e., $a_{c}=0.01 \lambda_{c}, c=1,2$ ). From Theorem 1 , if $D_{p}(c)$ denotes the weighted finite difference derivative approximation, then

$$
D_{p}(c)=\frac{2}{a_{j} T} \sum_{t=0}^{T-1} F_{c}\left(\lambda_{p}+a_{p} \sin (2 \pi \omega t)\right) \sin (2 \pi \omega t)=\frac{\partial}{\partial \lambda_{p}} F_{c}(\lambda)+\mathcal{O}\left(a_{p}^{2}\right) .
$$

The theoretical values for the system 1 experiments are

$$
F_{1}(\lambda)=2.1109, \quad F_{2}(\lambda)=3.8196
$$




$$
\begin{array}{rlr}
\frac{\partial}{\partial \lambda_{1}} F_{1}(\lambda) & =3.8017, & D_{1}(1)=3.8018 \\
\frac{\partial}{\partial \lambda_{1}} F_{2}(\lambda) & =16.3223, & D_{1}(2)=16.3250 \\
\frac{\partial}{\partial \lambda_{2}} F_{1}(\lambda) & =1.8182, & D_{2}(1)=1.8182 \\
\frac{\partial}{\partial \lambda_{2}} F_{2}(\lambda) & =11.3636, & D_{2}(2)=11.3638
\end{array}
$$

The theoretical values for the system 2 experiments are

$$
\begin{array}{rlrl}
F_{1}(\lambda) & =2.1109, & & F_{2}(\lambda)=3.8196 \\
\frac{\partial}{\partial \lambda_{1}} F_{1}(\lambda) & =1.4198, & & D_{1}(1)=1.4198 \\
\frac{\partial}{\partial \lambda_{1}} F_{2}(\lambda) & =2.3868, & & D_{1}(2)=2.3868 \\
\frac{\partial}{\partial \lambda_{2}} F_{1}(\lambda) & =1.1111, & D_{2}(1)=1.1111 \\
\frac{\partial}{\partial \lambda_{2}} F_{2}(\lambda) & =1.9753, & D_{2}(2)=1.9753
\end{array}
$$

Two-sided finite difference gradient estimates with CRN were also computed for the two sets of experiments. To ensure the same bias as the PHG estimator, the finite difference gradient estimates were obtained using $T=3$. These gradient estimates, with their associated root mean squared errors, for both systems, are given in Table 1.

\begin{tabular}{|c||c|c||c|c|}
\hline \multicolumn{1}{|c||}{} & \multicolumn{2}{c|}{$\mu=1.0, \lambda_{1}=0.45, \lambda_{2}=0.15$} & \multicolumn{2}{c|}{$\mu=1.0, \lambda_{1}=0.10, \lambda_{2}=0.15$} \\
\hline \hline & $\hat{D}_{p}(c)$ & $\mathrm{RMSE}$ & $\hat{D}_{p}(c)$ & $\mathrm{RMSE}$ \\
\hline$D_{1}(1)$ & 3.25 & 2.14 & 1.51 & 5.25 \\
$D_{1}(2)$ & 14.85 & 7.80 & 2.19 & 1.71 \\
$D_{2}(1)$ & 1.70 & 1.84 & 1.33 & 1.53 \\
$D_{2}(2)$ & 8.36 & 17.08 & 2.14 & 3.62 \\
\hline TRMSE & - & 18.99 & - & 6.78 \\
\hline
\end{tabular}

Table 1: Simulation Results for Finite Difference with CRN.

Table 2 and Table 3 provide computational results that compare the three methods for correlating the phantom systems, namely, all independent (IND), common random numbers (CRN) for systems $t$ and $T-t$, but independent phantoms for $t=1,2, \ldots, M$, and the combination of antithetic and common random numbers (ACRN), choosing $K$ independent random numbers and correlating the systems $t, T-t$ with $\mathrm{CRN}$ and $t, t+M$ with $\mathrm{ARN}, t+M, M+1-t$ with CRN, and $T-t, M+1-t$ with ARN, as depicted in Figure 2. The global efficiency measure TRMSE is reported in italics. 
The results obtained suggest several points. Using the PHG estimator with CRN yielded better results (as measured by the total root mean squared error) than the two-sided finite differences estimator with CRN and the PHG estimator with IND. Moreover, as $T$ increased, the total root mean squared error also decreased for all three PHG estimators (IND, CRN, and ACRN). However, the CPU times also increased with $T$, hence a balance between the quality of the resulting estimators with the computing time needed to obtain the estimates must be considered.

The results on the PHG estimator with ACRN were far less conclusive. In general, the results for the PHG estimator using either CRN or ACRN were comparable, particularly as $T$ increased. This is reasonable, since for $T$ small, the PHG estimator with ACRN cannot fully exploit the effect of ARN. However, using ACRN requires approximately half as many $U(0,1)$ variates, hence the CPU time necessary to obtain these results should be proportionately smaller. To fully exploit this efficiency may require a more extensive empirical study that focuses on the computational issues associated with implementing the PHG estimators. This is a topic of future research. Therefore, in light of these observations, there is no computational or estimation disadvantage in using ACRN rather than CRN.

\section{Conclusions}

This paper presents a new gradient estimator for the steady-state expected sojourn times for different customer classes in a nonpreemptive priority queueing system. The new estimator combines the phantom estimation method with the harmonic gradient estimator. The resulting estimator uses phantom systems to eliminate the bias and frequency selection problems associated with the harmonic gradient estimator, and can be computed using a single simulation run. This advantage increases as the number of priority classes $\mathrm{C}$ grows. Moreover, the unique form of the estimator allows both CRN and ARN streams to be simultaneously used as a variance reduction technique. Computational results with the estimator suggest that using CRN and ARN in this way can lead to a measurable variance reduction effect.

The variance of the PHG estimator is shown in Section 4 to be $\mathcal{O}(1 / N)$ for $N$ large and for any $T$. The PHG estimator variance analysis in Section 5 developed an upper bound expression for the conditional variance (given the nominal system trajectory), as well as showed that if the phantom systems are generated using independent Bernoulli sequences, then this conditional variance is $\mathcal{O}(1 / T)$ w.p.1 for $T$ large and for any $N$. Although the variance reduction technique using CRN and ARN for the Bernoulli sequences makes it difficult to undertake a more precise variance analysis, the variance analysis presented suggests that if the conditional covariance terms can be made negative, then the conditional variance expression in (16) can be further reduced. This expression is what motivated the design of the variance reduction technique using $\mathrm{CRN}$ and ARN, and provides 
anecdotal support for its application. Work is in progress to obtain a more complete understanding of how using both CRN and ARN impact the variance of the PHG estimator in particular, and gradient estimators in general.

The computational results suggest that the PHG estimator with CRN dominated both the two-sided finite difference estimators with CRN and the PHG estimator with IND, as measured by the total root mean squared error. Moreover, the results also suggest that if the PHG estimator with ACRN is efficiently implemented, the CPU time saving may justify the added effort in simultaneously using both CRN and ARN. See the appendix for a discussion on issues that affect the efficiency of implementing the PHG estimator. This is a topic of current research investigation.

\section{Acknowledgements}

The authors would like to thank the Simulation Area Editor, Dr. Michael Taaffe, and an anonymous referee for their insightful comments and valuable suggestions. Their feedback has resulted in a significantly improved paper.

\section{APPENDIX}

The implementation of parallel systems (programmed in C) defines a list for each phantom system. In computing all the derivatives, $T$ lists are needed for each derivative, resulting in a total of $2 T$ lists that must be defined. The main $\mathrm{C}$ program uses discrete-event simulation, where the simulation is driven by the nominal system, with the highest arrival rates $\lambda_{c}(1+a)$. Upon an arrival event, the class of customer, $j$, is identified and for each of the $T$ lists corresponding to index $j$, it is determined whether this arrival is a phantom customer according to the IND, CRN or ACRN rules. Once this is determined, the new arrival is always placed at the bottom of the list and a record is made that registers both the arrival time and the service time. This allows the CRNs to be synchronized by allocating the same service times to the individual customers in all the systems (where the service time is zero if the customer is a phantom in a given system). At the service events, however, a search is performed through all the lists to determine which customer has service in each of the phantom systems. Preliminary tests suggest that searching for the right ordering during service completions is more efficient then placing customers on the list when they arrive. Naturally, customers belonging to the same class will be served in the same order over all the systems, though swapping may occur between classes. Therefore, the $\mathrm{C}$ code developed was not designed to optimize execution by ranking comparisons and evaluating those events in the order in which they are most likely to occur (to avoid unnecessary Boolean operations) or by rewriting Lindley's equation for each customer class. 


\section{References}

[1] Andradóttir, S., 1998, "Simulation Optimization," in Handbook on Simulation, J. Banks, editor, Chapter 9, Wiley and Sons, New York, New York, 307-333.

[2] Brémaud, P., Vázquez-Abad, F.J., 1992, "On the Path-wise Computation of Derivatives with Respect to the Rate of a Point Process: the Phantom RPA Method," Queueing Systems, 10, 249-270.

[3] Cao, X.-R., 1985, "Convergence of Parameter Estimates in a Stochastic Experiment," IEEE Transactions on Automatic Control, AC-30:9, 845-853.

[4] Fu, M., 1994, "Optimization via Simulation: A Review," Annals of Operations Research, 53, 199-248.

[5] Glasserman, P., 1991, Gradient Estimation via Perturbation Analysis, Kluwer Academic Publishers, Boston, Massachusetts.

[6] Glasserman, P., Yao, D.D., 1992, "Some Guidelines and Guarantees for Common Random Numbers," Management Science, 38(6), 884-908.

[7] Gong, W.-B., 1988, "Smoothed Perturbation Analysis Algorithm for a GI/G/1 Routing Problem," Proceedings of the 1988 Winter Simulation Conference, M.A. Abrams, P.L. Haigh, J.C. Comfort, Editors, Piscataway, New Jersey, IEEE Press, 525-531.

[8] Gross, D., Harris, C.M., 1998, Fundamentals of Queueing Theory, Third Edition, Wiley and Sons, New York, New York.

[9] Jacobson, S.H., 1994, "Convergence Results for Harmonic Gradient Estimators," ORSA Journal on Computing, 6(4), 381-397.

[10] Jacobson, S.H., 1995, "How Difficult is the Frequency Selection Problem," Operations Research Letters, 17(3), 139-147.

[11] Jacobson, S.H., Buss, A.H., Schruben, L.W., 1991, "Driving Frequency Selection for Frequency Domain Simulation Experiments," Operations Research, 39(6), 917-924.

[12] Jacobson, S.H., Schruben, L.W., 1989, "Techniques for Simulation Response Optimization," Operations Research Letters, 8(1), 1-9.

[13] Jacobson, S.H., Schruben, L.W., 1999, "A Harmonic Analysis Approach to Simulation Sensitivity Analysis," IIE Transactions, 31(3), 231-243.

[14] Karlin, S., Taylor, H., 1975, A First Course in Stochastic Processes, Academic Press, New York, New York.

[15] Kushner, H.J., Vázquez-Abad, F.J., 1997, "Stochastic Approximation Methods for Systems over an Infinite Horizon," SIAM Journal on Control and Optimization, 34, 712-56. 
[16] Kushner, H.J., Yin, G., 1997, Stochastic Approximation and Applications, Springer Verlag, New York, New York.

[17] L'Ecuyer, P., 1990, "A Unified View of IPA, SF and LR Gradient Estimation Techniques," Management Science, 36(11), 1364-1383.

[18] Miyoshi, N., Hasegawa, T., 1995, "Smoothed Perturbation Analysis Estimates for a Stationary Multi-class Queue," Proceedings of the 34th Conference on Design and Control, 2612-2617.

[19] M.B. Priestley, 1989, Spectral Analysis and Time Series, Academic Press, London, England.

[20] Safizadeh, M.H., 1990, "Optimization in Simulation: Current Issues and the Future Outlook," Naval Research Logistics, 37, 807-825.

[21] Vázquez-Abad, F.J., 1999, "Strong Points of Weak Convergence: A Study Using RPA Gradient Estimation for Automatic Learning," Automatica, 35(7), 1255-1274.

[22] Vázquez-Abad, F.J., 2000 "A Course on Sensitivity Analysis for Gradient Estimation of DES Performance Measures", Part I of III, in Discrete Event Systems, Analysis and Control, R. Boel and G. Stremersch Editors, Kluwer Academic Publishers, Boston, Massachusetts, 3-28.

[23] Vázquez-Abad, F.J., Cassandras, C.G., Julka, V., 1998, "Centralized and Decentralized Asynchronous Optimization of Stochastic Discrete Event Systems," IEEE Trans on Automatic Control, 43(5), 631-655.

[24] Vázquez-Abad, F.J., L'Ecuyer, P., 1991, “Comparing Alternative Methods for Derivative Estimation when IPA Does Not Apply," Proceedings of the 1991 Winter Simulation Conference, B.L. Nelson, W.D. Kelton, G.M. Clark, Editors, Piscataway, New Jersey, IEEE Press, 10041011.

[25] Vázquez-Abad, F.J., Jacobson, S.H., 1994, "Application of RPA and the Phantom Harmonic Gradient Estimators to a Priority Queueing System," Proceedings of the 1994 Winter Simulation Conference, J.D. Tew, S. Manivannan, D.A. Sadowski, A.F. Seila, Editors, Piscataway, New Jersey, IEEE Press, 369-376.

[26] Zwillinger, D., 1992, Handbook of Integration, Jones and Bartlett Publishers, Boston, Massachusetts. 


\begin{tabular}{|c|c|c|c|c|c|c|}
\hline$T=5$ & \multicolumn{2}{|c|}{ Ind } & \multicolumn{2}{|c|}{$\mathrm{CRN}$} & \multicolumn{2}{|c|}{ ACRN } \\
\hline & $\hat{D}_{p}(c)$ & RMSE & $\hat{D}_{p}(c)$ & RMSE & $\hat{D}_{p}(c)$ & RMSE \\
\hline$D_{1}(1)$ & 3.72 & 2.66 & 4.10 & 2.39 & 3.65 & 2.03 \\
\hline$D_{1}(2)$ & 19.28 & 15.06 & 17.88 & 8.24 & 16.60 & 8.60 \\
\hline$D_{2}(1)$ & 2.09 & 2.78 & 2.03 & 2.02 & 1.58 & 1.39 \\
\hline$D_{2}(2)$ & 9.91 & 22.15 & 8.54 & 11.64 & 8.47 & 17.67 \\
\hline TRMSE & - & 27.06 & - & 14.60 & - & 19.81 \\
\hline CPU Time & \multicolumn{2}{|c|}{20} & \multicolumn{2}{|c|}{18} & \multicolumn{2}{|c|}{18} \\
\hline$T=9$ & \multicolumn{2}{|c|}{ Ind } & \multicolumn{2}{|c|}{$\mathrm{CRN}$} & \multicolumn{2}{|c|}{ ACRN } \\
\hline & $\hat{D}_{p}(c)$ & RMSE & $\hat{D}_{p}(c)$ & RMSE & $\hat{D}_{p}(c)$ & RMSE \\
\hline$D_{1}(1)$ & 3.89 & 1.51 & 3.77 & 1.50 & 3.64 & 1.56 \\
\hline$D_{1}(2)$ & 15.71 & 8.06 & 15.45 & 5.22 & 15.46 & 5.81 \\
\hline$D_{2}(1)$ & 1.55 & 1.05 & 1.86 & 1.29 & 1.59 & 1.28 \\
\hline$D_{2}(2)$ & 10.610 & 9.48 & 11.30 & 10.47 & 8.92 & 10.26 \\
\hline TRMSE & - & 12.58 & - & 11.87 & - & 11.96 \\
\hline CPU Time & \multicolumn{2}{|c|}{41} & \multicolumn{2}{|c|}{29} & \multicolumn{2}{|c|}{28} \\
\hline$T=17$ & \multicolumn{2}{|c|}{ IND } & \multicolumn{2}{|c|}{$\mathrm{CRN}$} & \multicolumn{2}{|c|}{ ACRN } \\
\hline & $\hat{D}_{p}(c)$ & RMSE & $\hat{D}_{p}(c)$ & RMSE & $\hat{D}_{p}(c)$ & RMSE \\
\hline$D_{1}(1)$ & 3.66 & 1.29 & 4.11 & 1.38 & 3.94 & 1.29 \\
\hline$D_{1}(2)$ & 16.87 & 6.37 & 16.91 & 6.32 & 17.17 & 5.50 \\
\hline$D_{2}(1)$ & 1.78 & 1.06 & 1.93 & 0.92 & 1.73 & 0.89 \\
\hline$D_{2}(2)$ & 11.90 & 10.57 & 10.45 & 8.42 & 12.69 & 9.95 \\
\hline TRMSE & - & 12.46 & - & 10.66 & - & 11.48 \\
\hline CPU Time & \multicolumn{2}{|c|}{56} & \multicolumn{2}{|c|}{50} & \multicolumn{2}{|c|}{47} \\
\hline
\end{tabular}

Table 2: Comparison of the Methods: System 1 


\begin{tabular}{|c|c|c|c|c|c|c|}
\hline$T=5$ & \multicolumn{2}{|c|}{ Ind } & \multicolumn{2}{|c|}{$\mathrm{CRN}$} & \multicolumn{2}{|c|}{ ACRN } \\
\hline & $\hat{D}_{p}(c)$ & RMSE & $\hat{D}_{p}(c)$ & RMSE & $\hat{D}_{p}(c)$ & RMSE \\
\hline$D_{1}(1)$ & 1.06 & 3.62 & 1.73 & 3.15 & 0.69 & 3.55 \\
\hline$D_{1}(2)$ & 2.16 & 1.40 & 2.20 & 1.56 & 2.23 & 1.72 \\
\hline$D_{2}(1)$ & 0.95 & .84 & 1.32 & 1.19 & 1.01 & 0.58 \\
\hline$D_{2}(2)$ & 1.73 & 2.82 & 1.76 & 2.18 & 1.67 & 2.58 \\
\hline TRMSE & - & 4.87 & - & 4.15 & - & 4.75 \\
\hline CPU Time & \multicolumn{2}{|c|}{14} & \multicolumn{2}{|c|}{12} & \multicolumn{2}{|c|}{12} \\
\hline$T=9$ & \multicolumn{2}{|c|}{ Ind } & \multicolumn{2}{|c|}{$\mathrm{CRN}$} & \multicolumn{2}{|c|}{ ACRN } \\
\hline & $\hat{D}_{p}(c)$ & RMSE & $\hat{D}_{p}(c)$ & RMSE & $\hat{D}_{p}(c)$ & RMSE \\
\hline$D_{1}(1)$ & 1.10 & 3.26 & 1.77 & 2.75 & 0.87 & 2.41 \\
\hline$D_{1}(2)$ & 2.20 & 1.24 & 2.28 & 1.03 & 2.24 & 1.08 \\
\hline$D_{2}(1)$ & 1.12 & 0.60 & 1.14 & 0.76 & 0.99 & 0.48 \\
\hline$D_{2}(2)$ & 1.59 & 1.98 & 1.96 & 2.04 & 2.06 & 1.71 \\
\hline TRMSE & - & 4.06 & - & 3.66 & - & 3.18 \\
\hline CPU Time & \multicolumn{2}{|c|}{22} & \multicolumn{2}{|c|}{19} & \multicolumn{2}{|c|}{22} \\
\hline$T=17$ & \multicolumn{2}{|c|}{ IND } & \multicolumn{2}{|c|}{$\mathrm{CRN}$} & \multicolumn{2}{|c|}{ ACRN } \\
\hline & $\hat{D}_{p}(c)$ & RMSE & $\hat{D}_{p}(c)$ & RMSE & $\hat{D}_{p}(c)$ & RMSE \\
\hline$D_{1}(1)$ & 1.52 & 2.10 & 1.42 & 2.06 & 0.99 & 2.18 \\
\hline$D_{1}(2)$ & 2.47 & 1.10 & 2.23 & 0.66 & 2.34 & 0.93 \\
\hline$D_{2}(1)$ & 1.06 & 0.42 & 1.13 & 0.52 & 1.16 & 0.55 \\
\hline$D_{2}(2)$ & 2.22 & 1.52 & 2.03 & 1.55 & 1.96 & 1.53 \\
\hline TRMSE & - & 2.85 & - & 2.71 & - & 2.87 \\
\hline CPU Time & \multicolumn{2}{|c|}{39} & \multicolumn{2}{|c|}{33} & \multicolumn{2}{|c|}{28} \\
\hline
\end{tabular}

Table 3: Comparison of the Methods: System 2 